\section{P293 IS OBSTRUCTIVE SLEEP APNOEA A RISK FACTOR FOR CHRONIC KIDNEY DISEASE?}

${ }^{1} \mathrm{~F}$ Rauf, ${ }^{1} \mathrm{~J}$ Kerks, ${ }^{1} \mathrm{D}$ Comer, ${ }^{1} \mathrm{I}$ Dasgupta, ${ }^{1} \mathrm{M}$ Daniels, ${ }^{2} \mathrm{R}$ Mukherjee, ${ }^{1} \mathrm{~S}$ Wharton. ${ }^{1}$ Birmingham Heartlands Hospital, Birmingham, UK; ${ }^{2}$ School of Clinical andExperimental Medicine, University of Birmingham, Birmingham, UK

\subsection{6/thoraxjnl-2014-206260.411}

Background Obstructive sleep apnoea (OSA) is associated with intermittent hypoxaemia which leads to activation of a number of pathways including oxidative stress, sympathetic nervous system, endothelial dysfunction and inflammation. These in turn lead to hypertension and atherosclerosis. There is some evidence that the same process may predispose to renal dysfunction. In this retrospective study, we compared a group people with hypertension and OSA on continuous positive airways pressure therapy (CPAP) with matched control group not known to have OSA.

Method Patients with known OSA and controls matched for age, sex and BMI were selected retrospectively from a hypertension clinic database. The two groups were compared using the following parameters: mean 24-hour systolic blood pressure (SBP, $\mathrm{mmHg}$ ), mean serum creatinine (micromol/l) and mean urine albumin/creatinine ratio (ACR. $\mathrm{mg} / \mathrm{mmol}$ ).

Results Forty-nine patients were identified with confirmed OSA on CPAP. 6 were excluded due to insufficient data. Of the 43 remaining patients 35 were male, the mean age was 53.5 years and the mean BMI was $34.6 \mathrm{~km} / \mathrm{m}^{2}$. The mean SBP was 143 in patients versus 135 in controls $(\mathrm{p}=0.04)$. The mean serum creatinine was 94.0 in patients versus 93.4 in controls $(\mathrm{p}=0.45)$. The mean ACR was 2.50 in patients versus 0.63 in controls ( $p$ $=0.031$ ).

Conclusion This retrospective case-control study shows a higher prevalence of hypertension and proteinuria in patients with treated OSA when compared with controls matched for age, sex and BMI. OSA in the patients may not have been adequately treated. There may have been undiagnosed OSA in the controls. Patients' higher proteinuria may have been caused by their more severe hypertension. Causation is not proven. However this study raises the possibility that OSA might predispose to chronic kidney disease. A larger, prospective study might confirm this finding and provide information on causation.

\section{P294 FACTORS AFFECTING CPAP COMPLIANCE}

JA Stockley, S Huq, S Madathil, JA Hunt, BG Cooper. Queen Elizabeth Hospital, Birmingham, UK

\subsection{6/thoraxjnl-2014-206260.412}

Rationale Due to the nature and sensation of CPAP, not all patients comply with the treatment. Factors such as age, degree of sleepiness, health status, BMI and sleep apnoea severity may influence CPAP adherence. We sought to determine if these and other factors, particularly socioeconomic status, influence CPAP compliance of our patients.

Methods Demographic data (including age, sex, race and BMI), Epworth Sleepiness Score, Oxygen Desaturation Index (ODI; Desaturations/hour $>4 \% \mathrm{SpO}_{2}$ ) and prescribed CPAP were retrospectively collected from all patients who had been issued CPAP between 2009 and 2013. In addition, the Index of Multiple Deprivation (IMD) Score and Rank were retrieved from the
Abstract P294 Table 1 Statistical comparisons between CPAP compliers and non-compliers. A better socioeconomic status is indicated by a lower IMD Score and a higher IMD Rank value. Age is presented as the median (range), sex and race are presented as\% dominant trait and all other data are presented as median (IQR)

\begin{tabular}{llll}
\hline Demographic & Compliers $(\mathrm{n}=287)$ & Non-Compliers $(\mathrm{N}=120)$ & $\mathrm{p}$ value \\
\hline Age (years) & $51(20-84)$ & $52(24-76)$ & $\mathrm{ns}$ \\
$\%$ Male & 80.5 & 72.5 & $\mathrm{~ns}$ \\
\% Caucasian & 82.2 & 82.5 & $\mathrm{~ns}$ \\
BMI $\left(\mathrm{kg} / \mathbf{m}^{2}\right)$ & $37.2(32.2,42.1)$ & $34.6(29.9,40.2)$ & 0.02 \\
Epworth & $14(11,17)$ & $12(9,17)$ & 0.02 \\
ODI & $20.9(16.1,57.8)$ & $19.9(10.8,45.2)$ & $<0.01$ \\
CPAP $\left(\mathrm{cmH}_{2} \mathbf{0}\right)$ & $12(10,12)$ & $11(10,12)$ & $\mathrm{ns}$ \\
IMD Score & $25.2(15.3,44.2)$ & $29.2(17.6,47.9)$ & $\mathrm{ns}$ \\
IMD Rank & $10527(3344,17818)$ & $8695(2594,15936)$ & $\mathrm{ns}$ \\
\hline
\end{tabular}

www.neighbourhood.statistics.gov.uk website. These parameters were then compared between CPAP "compliers" $(\geq 4 \mathrm{hrs} /$ night and $\geq 4$ nights/week) and "non-compliers" ( $<4$ hrs/night or $<4$ nights/week).

Results We obtained complete data from 407 patients over the four years period. Compliance was achieved in $70.5 \%$ of patients. CPAP compliers had significantly worse sleep apnoea (ODI), degree of sleepiness (Epworth) and a higher BMI (Table $1)$

Conclusions Our data show a good level of compliance compared to previous reports (Shapiro et al. Sleep Breath 2010; 14: 323-25) and patient adherence at 12 months is better than previously reported (Bollig. Respir Care 2010; 55: 1230-9). Although ODI, Epworth and BMI were significantly higher in the CPAP compliers, the differences were only modest. Among the demographics tested, none seem to have a major influence on CPAP compliance in our cohort of patients.

\section{P295 PATIENTS' PREFERENCE OF ESTABLISHED AND EMERGING TREATMENTS FOR OBSTRUCTIVE SLEEP APNOEA}

${ }^{1} \mathrm{~T}$ Campbell, ${ }^{2} \mathrm{MF}$ Pengo, ${ }^{3} \mathrm{R}$ Brown, ${ }^{3} \mathrm{~A}$ Birdseye, ${ }^{3} \mathrm{~K}$ Bacon, ${ }^{4} \mathrm{~J}$ Steier. ${ }^{1}$ Georgetown University School of Medicine, Washington, DC, USA; ${ }^{2}$ Department of Medicine (DIMED), University of Padua, Italy; ' Guy's and St. Thomas' NHS Foundation Trust, Lane Fox Respiratory Unit/Sleep Disorders Centre, London, UK; ${ }^{4}$ King's College London School of Medicine, London, UK

\subsection{6/thoraxjnl-2014-206260.413}

Background Obstructive sleep apnoea (OSA) is the most common form of sleep-disordered breathing, and the standard treatment is continuous positive airway pressure (CPAP). Emerging treatments for OSA, including electrical hypoglossal nerve stimulation (HNS) and non-invasive electrical stimulation (nES), are currently being developed. To involve patients in the development of research projects, we evaluated patients' preference for different treatments of OSA using a short survey.

Patients and methods We recorded patients' age, gender, bodymass-index (BMI), Epworth Sleepiness Scale (ESS), Functional Outcomes of Sleep Questionnaire (FOSQ10), severity of OSA, and current treatment. We showed pictures of existing (CPAP, mandibular advancement device (MAD)) and emerging treatments (HNS and nES). We then asked 1) whether they were 